\title{
Adapted Transfer Function Design for Coronary Artery Evaluation
}

\author{
Sylvia Glaßer ${ }^{1}$, Steffen Oeltze ${ }^{1}$, Anja Hennemuth ${ }^{2}$, Skadi Wilhelmsen ${ }^{3}$, \\ Bernhard Preim ${ }^{1}$ \\ ${ }^{1}$ Department of Simulation and Graphics, University of Magdeburg \\ ${ }^{2} \mathrm{MeVis}$ Research $\mathrm{GmbH}$, Bremen \\ ${ }^{3}$ Centre of Radiology, University Hospital, Magdeburg \\ glasser@isg.cs.uni-magdeburg.de
}

\begin{abstract}
We present an approach for adapted transfer function (TF) specification to highlight pathologic changes of the coronary artery vessel wall based on contrast medium-enhanced computed tomography (CT) datasets. The TFs are combined with common visualization techniques for the evaluation of coronary arteries in CT image data. The presented mapping of $\mathrm{CT}$ values in Hounsfield Units (HU) to optical properties such as color and opacity leads to a different color coding for different plaque types.
\end{abstract}

\section{Introduction}

Coronary artery disease (CAD) is the leading cause of death in western nations [1]. Recent developments in multislice spiral computed tomography (MSCT) allow for a high spatial resolution with short acquisition times and thus for noninvasive evaluation of the coronary arteries. In contrast to conventional angiograms, MSCT image data also enable an assessment of the vessel wall's cross-sectional area. Furthermore, MSCT image data have a great potential for noninvasive identification, characterization, and quantification of atherosclerotic CAD [2].

Atherosclerotic CAD is the result of accumulatations in the artery wall, socalled plaques. They can be classified into soft, fibrous and hard plaques. Soft plaques are prone to rupture and thus very dangerous for the patient, whereas fibrous plaques and hard plaques consist of more dense accumulations and are assumed to be more stable. Hard plaques are characterized by calcium deposits. The quantification of coronary calcium is important in assessing the patient's whole plaque burden. A plaque does not necessarily lead to a significant stenosis. Especially the early states of atherosclerotic CAD can be compensated by a positive remodelling of the vessel wall.

Common strategies for the visualization of the coronary artery tree based on MSCT image data are maximum intensity projection (MIP), multiplanar reformation (MPR), curved multiplanar reformation (CPR) for $2 \mathrm{D}$ views and direct volume rendering techniques (DVR) for a $3 \mathrm{D}$ view $[2,3,4]$. The mapping 
from CT values in Houndsfield Units (HU) to optical properties such as color and opacity is described by a transfer function (TF). The windowing function, which is a special instance of a TF, maps an interval of $\mathrm{HU}$ values to gray values. The user can manipulate the interval's width and center and thus influence brightness and contrast.

We will present visualization techniques that facilitate a visual separation of hard plaques and highlight the coronary artery wall including soft and fibrous plaques. In contrast to the method presented by Wesarg et. al [5] we also account for non-stenotic plaque deposits. Due to varying HU values of the bloodpool caused by the contrast medium, the TFs have to be adapted to each dataset.

\section{Materials and Methods}

We developed our techniques based on 12 contrast medium-enhanced MSCT coronary angiograms from a 64-slice scanner (Siemens Sensation 64). For each dataset the segmentation of the coronary artery lumen [6] and the skeleton of the segmentation result were available. For the TF specification the mean density and standard derivation of the bloodpool $\left(\mu_{\text {blood }}, \sigma_{\text {blood }}\right)$ and the vessel wall $\left(\mu_{\text {wall }}, \sigma_{\text {wall }}\right)$ have to be approximated.

\subsection{Approximation of blood density values}

The segmentation result primarily consists of voxels representing the contrast medium-enhanced blood and is used for the approximation of the blood density distribution. Because of other densities originating from surrounding tissue or interpolation issues, $\mu_{\text {blood }}$ and $\sigma_{\text {blood }}$ could not be directly derived from the segmentation. The approximation therefore is carried out by means of an iterative reduction of a cost function. Costs are defined as differences between the approximated normal distribution and the distribution of all segmented voxels. Results for $\mu_{\text {blood }}$ and $\sigma_{\text {blood }}$ indicate that the mean density of the blood strongly differs for each dataset. Thus, a static threshold for a separation of hard plaques from blood is not applicable. We use a threshold $s$, similar to the threshold of the Agatston score [7]. To avoid overestimation, which may arise due to artifacts or contrast medium accumulation in small branches, we define $s$ as $s=\mu_{\text {blood }}+3 \sigma_{\text {blood }}$.

\subsection{Approximation of vessel wall density values}

The approximation of the vessel wall density values is based on the evaluation of a coronary artery segment and its skeleton. The vessel wall of this segment is analyzed by means of intensity profile volumes (IPV). The IPV calculation is carried out in 4 steps (Fig. 1):

1. Selection of a long, non-branching segment of the coronary artery tree. The selection is carried out by traversing the skeleton of the segmentation. 
2. The real vessel centerline is approximated by the segment skeleton.

3. For each skeleton voxel, $n$ rays perpendicular to the skeleton are casted.

4. The rays are sampled and the sampled intensities are saved in the IPV.

After the IPV extraction, the vessel wall densities can be approximated. For each skeleton voxel, the sampled intensities representing the vessel wall will appear as vertical structures in a slice of the IPV (Fig. 1(b)). The IPV is slicewise convoluted with a Gaussian and a Sobel filter. The convolutions are followed by a search for vertical structures to obtain a sample of vessel wall intensities. Based on this sample, $\mu_{\text {wall }}$ and $\sigma_{\text {wall }}$ is calculated.

\section{$2.3 \quad$ TF specification}

We developed two TFs, the $T F_{2 D}$ and the $T F_{3 D}$, for $2 \mathrm{D}$ and $3 \mathrm{D}$ visualizations. They differ only in the interval size of the vessel wall densities. We use $\mu_{\text {wall }} \pm$ $\sigma_{\text {wall }}$ for the $T F_{3 D}$ and $\mu_{\text {wall }} \pm 2 \sigma_{\text {wall }}$ for the $T F_{2 D}$. This is motivated by a possible occlusion of inner structures of the vessel wall in $3 \mathrm{D}$ visualizations. Therefore, we also use smaller opacity values for the vessel wall for the $T F_{3 D}$ than for the $T F_{2 D}$. In comparison, the vessel wall and hard plaques are mapped to higher opacity values, whereas absolute transparency is assigned to the surrounding tissue and the contrast medium-enhanced blood.

We intuitively use colors from beige to white for hard plaques, since these structures usually appear white or light gray in conventional CT-displays. For the vessel wall, we employ a color scale from blue over red to green to provide high contrasts for the visualization of different plaque deposits and thus different densities in the vessel wall.

For a more intuitive view, the $T F_{2 D}$ is combined with the windowing $T F$. The user can now choose interesting $\mathrm{HU}$ value intervals by manipulating the parameters of the windowing TF. The CPR and MPR view as well as the 3D spatial variation of the coronary artery tree are linked with each other. On the one

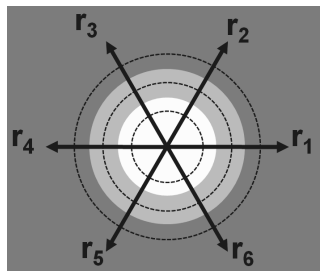

(a)

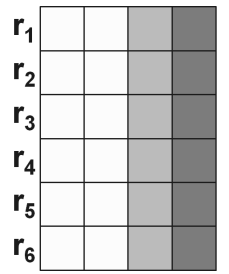

(b)

Fig. 1. Extraction of a slice of the intensity profile volume (IPV). For each voxel $v$ of the skeleton of a vessel segment, $n$ (e.g. 6) rays perpendicular to the centerline are casted (a). Along the rays, intensities are sampled and saved in a slice of the IPV (b). A cross-section of an ideal vessel (white) and the vessel wall (light gray) with surrounding tissue (dark gray) leads to vertical structures in the IPV slice for $v$. The IPV has as many slices as the number of centerline voxels. 
hand, the user can traverse the CPR view with the correspondending MPR view being updated. On the other hand, it is possible to pick an interesting vessel part in the $3 \mathrm{D}$ view with the other views being updated accordingly. The presented techniques have been integrated in the MeVisLab platform (www.mevislab.de).

\section{Results}

For each of the 12 datasets, we created adapted TFs based on the specific parameters $\mu_{\text {blood }}, \sigma_{\text {blood }}, \mu_{\text {wall }}$ and $\sigma_{\text {wall }}$. We combined the adapted TFs with common 2D and 3D visualizations (Fig. 2). Our method highlights not only stenotic plaque deposits, but also displays smaller plaques without a significant stenosis.

$3 \mathrm{D}$ visualizations show the spatial variation of the coronary artery tree and indicate the patient's whole plaque burden by highlighting all hard plaques (Fig. 2a). In the combined CPR-MPR view, the plaques are highlighted with different colors depending on the plaque type (Fig. 2b).

The methods and visualizations were discussed with an experienced radiologist. First comparisons between conventional views and the $T F_{2 D}$ and $T F_{3 D}$ color coded visualizations indicate, that hard plaques, especially smaller ones, can be better detected in the colored view. Furthermore, the color coding of the vessel wall with different colors for fibrous and soft plaque deposits gives indications about the plaque type. An absolute identification is impossible due to overlapping density intervalls of these types [8].

\section{Discussion}

We presented a method for the visualization of the coronary artery wall and atherosclerotic plaque based on adapted TFs. Our method highlights hard plaques and soft and fibrous plaques by color coding of the vessel wall. Also early stages of the CAD consisting of non-stenotic plaque deposits can be visualized. The adapted TFs were combined with common visualization techniques of CT coronary angiograms. An informal evaluation with one radiologist revealed that our method is a useful extension for conventional CT coronary angiogram evaluation.

Problems arise due to the local vessel wall density approximation. Falsifications in MPR views of other segments could be observed in 2 datasets. Potential improvements are the individual creation of a local TF for each vessel segment or a global approximation of the vessel wall density. The highlighting of dense structures such as hard plaques leads to the highlighting of coronary stents as well. A further improvement of the visualization could be the segmentation of stents in a preprocessing step.

Acknowledgement. We thank Dr. Stephan Achenbach (University of Erlangen-Nürnberg, Germany) for providing the image data and MeVis Research for providing advanced MeVisLab features. 


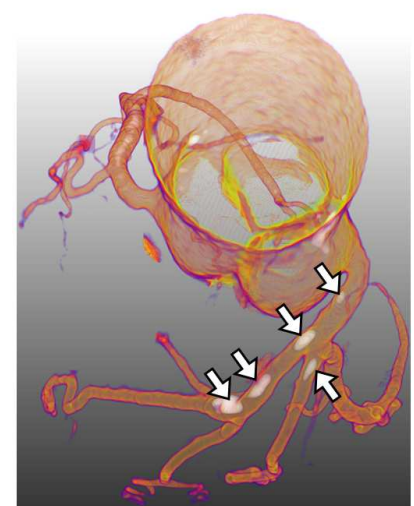

(a)
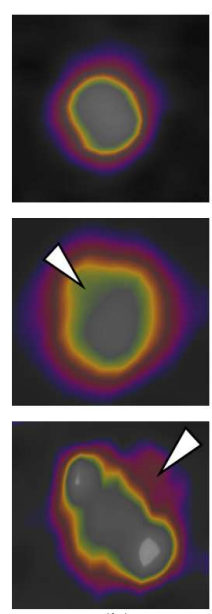

(b)

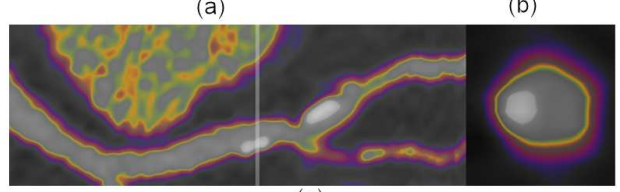

(c)

\section{References}

1. American Heart Association. Heart disease and stroke statistics - 2007 update. Circulation. 2007;115:e69-e171.

2. Schoepf UJ, Becker CR, Ohnesorge BM, et al. CT of coronary artery disease. Radiology. 2004;232(1):18-37.

3. Kanitsar A, Fleischmann D, Wegenkittl R, et al. CPR - curved planar reformation. Proc IEEE Vis. 2002; p. 37-44.

4. Straka M, Cervenansky M, Cruz AL, et al. The VesselGlyph: Focus \& context visualization in CT-angiography. Proc IEEE Vis. 2004; p. 385-392.

5. Wesarg S, Fawad Khan M, Firle EA. Localizing calcifications in cardiac CT data sets using a new vessel segmentation approach. J Digit Imaging. 2006;19(3):249-257.

6. Hennemuth A, Boskamp T, Fritz D, et al. One-click coronary tree segmentation in CT angiographic images. LNCS. 2005;1281:317-21.

7. Agatston AS, Janowitz WR, Hildner FJ, et al. Quantification of coronary artery calcium using ultrafast computed tomography. J Am Coll Cardiol. 1990;15(4):827832.

8. Pohle K, Achenbach S, MacNeill B, et al. Characterization of non-calcified coronary atherosclerotic plaque by multi-detector row CT: Comparison to IVUS. Atherosclerosis. 2007;190:174-180. 